\title{
Detailed Measurements of Boundary Layer Flow under Swash with High-resolution PIV
}

\author{
Dejun FENG ${ }^{1}$, Lianhui WU2 ${ }^{2}$, Takenori SHIMOZONO 3 and Akio OKAYASU4 \\ ${ }^{1}$ Graduate School of Marine Science and Technology, Tokyo University of Marine Science and Technology \\ (4-5-7, Konan, Minato-ku, Tokyo 108-0075, Japan) \\ E-mail: d132016@kaiyodai.ac.jp \\ ${ }^{2}$ Graduate School of Marine Science and Technology, Tokyo University of Marine Science and Technology \\ (4-5-7, Konan, Minato-ku, Tokyo 108-0075, Japan) \\ E-mail: m124028@kaiyodai.ac.jp \\ ${ }^{3}$ Member of JSCE, Assistant professor, Dept. of Civil Eng., University of Tokyo \\ (7-3-1, Hongo, Bunkyo-ku, Tokyo 113-8656, Japan) \\ E-mail: shimozono@coastal.t.u-tokyo.ac.jp \\ ${ }^{4}$ Member of JSCE, Professor, Dept. of Ocean Sciences, Tokyo University of Marine Science and Technology \\ (4-5-7, Konan, Minato-ku, Tokyo 108-0075, Japan) \\ E-mail: okayasu@kaiyodai.ac.jp
}

\begin{abstract}
A laboratory study under swash flow on a steep slope was designed to investigate the characteristics of bottom boundary layer. By careful setup of the PIV system and optimizing the traditional cross-correlation algorithm, the velocity fields within this thin boundary layer of an order of $1 \mathrm{~mm}$ were successfully resolved over ten points. The spatially and temporally resolved near-bed velocity profiles had higher resolution than previous studies and were expected to make reliable insights into the boundary layer structure, including the mean velocities, turbulence intensity and bed shear stress. Results suggested that mean velocities and turbulence intensity in uprush are larger than that in the backwash. The uprush flow is associated with the bore-generated and bed-generated turbulence, while the backwash flow is supposed to be dominated by bed-generated turbulence, and the high turbulence intensity in the final stage of backwash is supposed to be related to high velocity gradient. The bed shear stress estimated by fitting the logarithmic profiles to the measured velocity profiles in the uprush was larger than that in the backwash. The detailed measurement data can be used to develop and verify numerical models for boundary layer studies under swash.
\end{abstract}

Key Words: boundary layer, swash zone, PIV, turbulence intensity, bed shear stress

\section{INTRODUCTION}

The swash zone, where flows run up and wash down the beach face, is widely recognized as the most dynamic region in coastal zone with active sediment transport. The flow in this region, induced by waves of different frequencies, wave breaking and swash-swash interaction is highly unsteady and non-uniform.

The flow characteristics in this thin and aerated region have not been well understood quantitatively, especially those within thin boundary layers. In the past decade, a number of field and laboratory investigations have been attempted to investigate the swash zone hydrodynamics using various flow visualization techniques. Cowen ${ }^{1)}$ et al. made the first attempt to investigate the vertical flow structures in wave-driven swash zone using PIV. They found the turbulence characteristics of swash flows are very different during uprush and backwash. The uprush is well characterized as decaying grid turbulence, while backwash is represented by a temporally growing wall boundary layer flow. Recently, O'Donoghue ${ }^{2)}$ et al. conducted new laboratory experiments to measure the detailed hydrodynamics in bore-driven swash zone on a steep beach. Excellent repeatability of their facility made it possible to deeply investigate the turbulence characteristics using ensemble averaging method. It was demonstrated that the turbulence intensity in the uprush is larger than that in the backwash and the depth-averaged turbulence intensity is typically an order of magnitude greater on rough beaches compared to smooth beds.

However, a common problem of the previous la- 


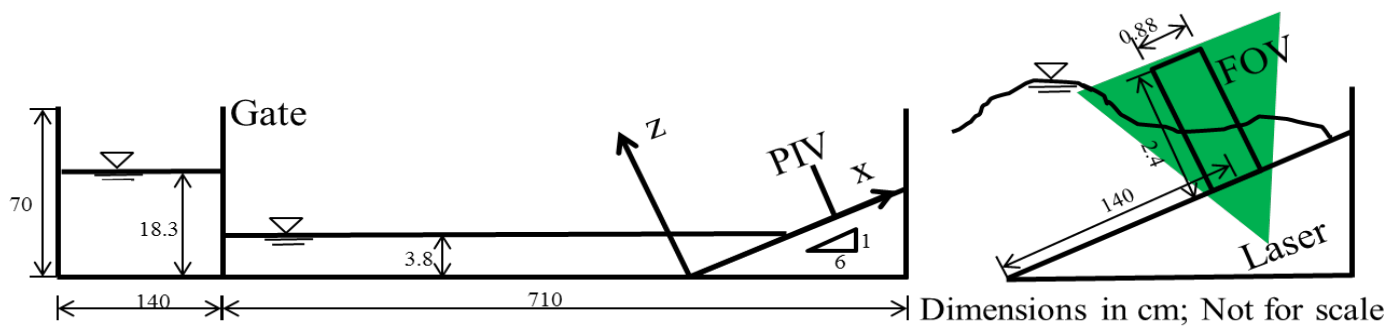

Fig.1 Experimental setup and the enlarged view of the measurement section.

boratory studies was that their measurements were not done sufficiently close to the bed and thus the corresponding discussions still contain a large amount of uncertainty. This is largely due to difficulties in flow measurements in the thin layer of transient water. The flow processes within the bottom boundary layer cannot be best studied without accurate and detailed measurements there. The primary purpose of the present study is to measure velocity profiles inside the bottom boundary layer under dambreak-generated swash using enhanced high-resolution PIV technique. This is a vital step forward to test and improve the modeling capability of the boundary layer flow in the swash zone.

\section{EXPERIMENTAL SETUP}

(1) Facility and instrumentation

The experiments were carried out using an open channel flume. It is an $8.5 \mathrm{~m}$ long, $0.3 \mathrm{~m}$ wide and 0.7 $\mathrm{m}$ high, glass-sided flume with a water reservoir built into one end as sketched in Fig.1. A 1/6 transparent slope made of acylics with a thickness of $8 \mathrm{~mm}$ was constructed at the other end of the flume. The reservoir is fronted by a gate which can be raised to produce a large plunging wave leading to a bore that propagates towards the slope. The overall swash flow induced by the single bore wave is simpler without interactions between uprush and backwash caused by successive waves. Moreover, the longer bore-generated swash motion enables longer velocity measurements. Velocities near the bottom on the slope were measured at the location $140 \mathrm{~cm}$ landward from the toe of the slope by a high-speed CCD camera (Detect HAS-500) with $2000 \mathrm{fps}, 1 / 4000 \mathrm{~s}$ shutter speed, $600 \times 220$ pixels field of view (FOV). The camera was rotated to be aligned with the slope to get the bed-parallel and bed-normal velocities. A continuous laser system, which has a wavelength of $532 \mathrm{~nm}$, an energy output of $1 \mathrm{~W}$ and a light sheet thickness of $1 \mathrm{~mm}$, was installed below the slope to illuminate the measuring section upward to avoid
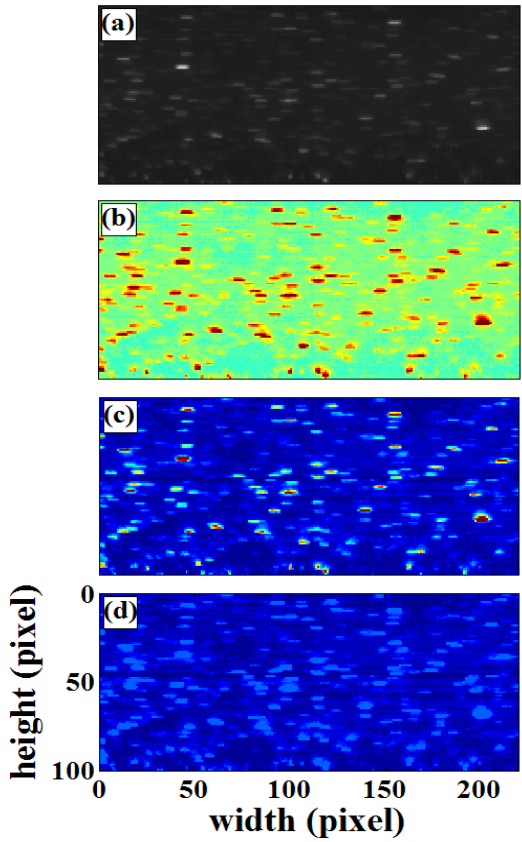
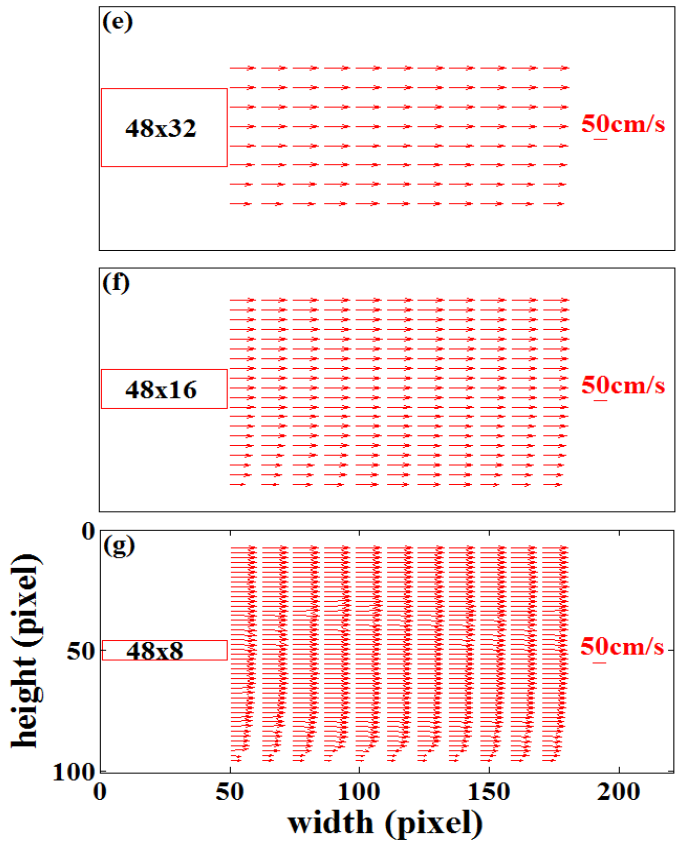

Fig.2 (a) raw image; (b) raw image displayed with color; (c) background-subtracted image; (d) intensity capped image; (e) 48 $\times 32$; (f) $48 \times 16$; (g) $48 \times 8$; the red rectangles in e, f, $g$ are the corresponding interrogation windows. 


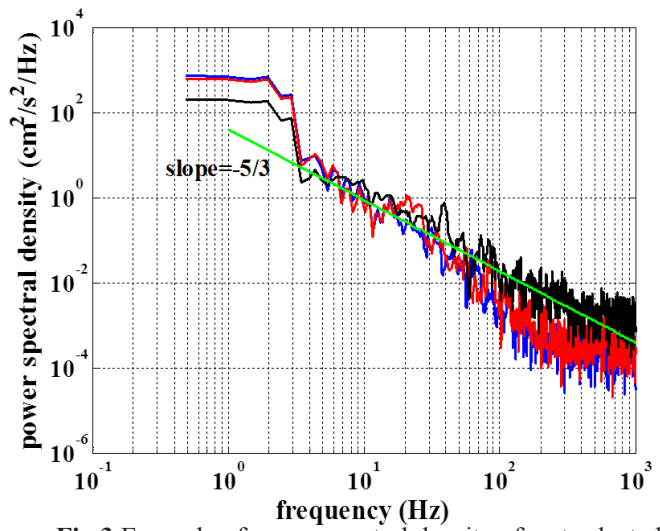

Fig.3 Example of power spectral density of $u$ at selected elevations above the bed. Blue, $3.6 \mathrm{~mm}$; red, $1.96 \mathrm{~mm}$; black, $0.24 \mathrm{~mm}$. The green line indicates the $-5 / 3$ slope.

free surface disturbances. The laser beam was about $2.5 \mathrm{~cm}$ apart from the nearest sidewall. Glass beads with a mean diameter of $60 \mu \mathrm{m}$ were used as tracer particles. The specific gravity is slightly larger than unity and thus it follows the flow well. Average concentration of the particle in water was optimized to $0.08 \mathrm{~g} / 1$ through preliminary tests. The camera was positioned at the minimum distance in the focal range to the laser beam for a large image magnification; thereby only the bottom part of the water depth was imaged with a spatial resolution of $40 \mu \mathrm{m} /$ pixel.

The origin of the $x-z$ coordinate system is set at the toe of the slope with the $x$-axis parallel to the slope and the $z$-axis perpendicular to the slope. The corresponding velocity components are denoted by $u$ and $w$, respectively. The experiments were conducted for one initial condition only: the initial water depth of $183 \mathrm{~mm}$ in the reservoir and $38 \mathrm{~mm}$ on the flume floor. The water level setting was determined for the longest swash motion prorogation in the current experimental facility. The experiment was repeated 100 times under the same condition. However, the repeatability of the swash flow was found not good enough to employ ensemble averaging method. Therefore, the analysis is based on the data of one representative run.

\section{(2) PIV images processing}

Only $220 \times 100$ pixels (the highest measurement elevation is 220 pixels above the bed) were used in the images for the velocity calculation, since our focus is on the bottom boundary layer. The procedure of image processing was illustrated in Fig.2. Firstly, a background image was removed by subtracting the minimum intensity image from each raw recording to remove the effects of stationary image features. Then, Intensity Capping Method ${ }^{3}$, which sets intensities larger than a preset threshold to the threshold value, was used to bring image intensities of all the tracer particles to a similar signal level. After that the instantaneous velocity field was calculated based on an Adaptive Cross-Correlation algorithm, with three steps using different interrogation windows and a $75 \%$ overlap: $48 \times 32,48 \times 16$, and $48 \times 8$. Integer displace information in the previous step was used to offset the interrogation window in the next step as outlined by Westerweel ${ }^{4}$ et al. The three-step approach raises the vertical measurement resolution without increasing mismatch caused by a small interrogation window. Note that, the interrogation window in the present study was a horizontally long rectangle rather than a square used in most previous studies. There are two main reasons for this setting: i) it can increase the vertical resolution more significantly compared with a square; ii) it can decrease the negative impacts of vertical gradients on the correlation since the shear is recognized as a significant feature in the bottom boundary layer as explained by PLF $\mathrm{Liu}^{5}$ ) et al. Spurious vectors were detected and replaced by their local median through by the Normalized Median Test $^{6}$ ). The final data set contains $11 \times 45$ velocity vectors in each image with a spatial resolution of $0.08 \mathrm{~mm}$ in the $z$ direction and $0.48 \mathrm{~mm}$ in the $x$ direction. Fig. 2 shows an example instantaneous velocity field when the water depth reaches $4 \mathrm{~mm}$ during the uprush. As shown in the figure, velocity profiles within the boundary layer with a thickness of around $1 \mathrm{~mm}$ (about 25 pixels) were successfully captured using the high-resolution PIV system. This is of great advantage over previous studies, whose measurements were limited to flow depth greater than approximately $10 \mathrm{~mm}$.

\section{(3) Velocity data analysis}

The measured instantaneous velocity vectors along the centerline of each image were defined as the representative velocity in the measurement window and used for further analysis. Separation of mean and turbulent velocity components from a single experiment in strongly unsteady and nonlinear flow conditions is a tough task. The most frequently used method, which makes a numerical filtering of the measured data in the frequency domain 7 ), was utilized in this study. The cut-off frequency was decided by checking the spectral slope of measured velocity data. Fig.3 shows the computed power spectral density of $u$ at three vertical elevations of 3.6 $\mathrm{mm}, 1.96 \mathrm{~mm}$ and $0.24 \mathrm{~mm}$ above the bed. The spectral slope in the around 5 to $30 \mathrm{~Hz}$ frequency range is close to the value of $-5 / 3$, which is typical of 

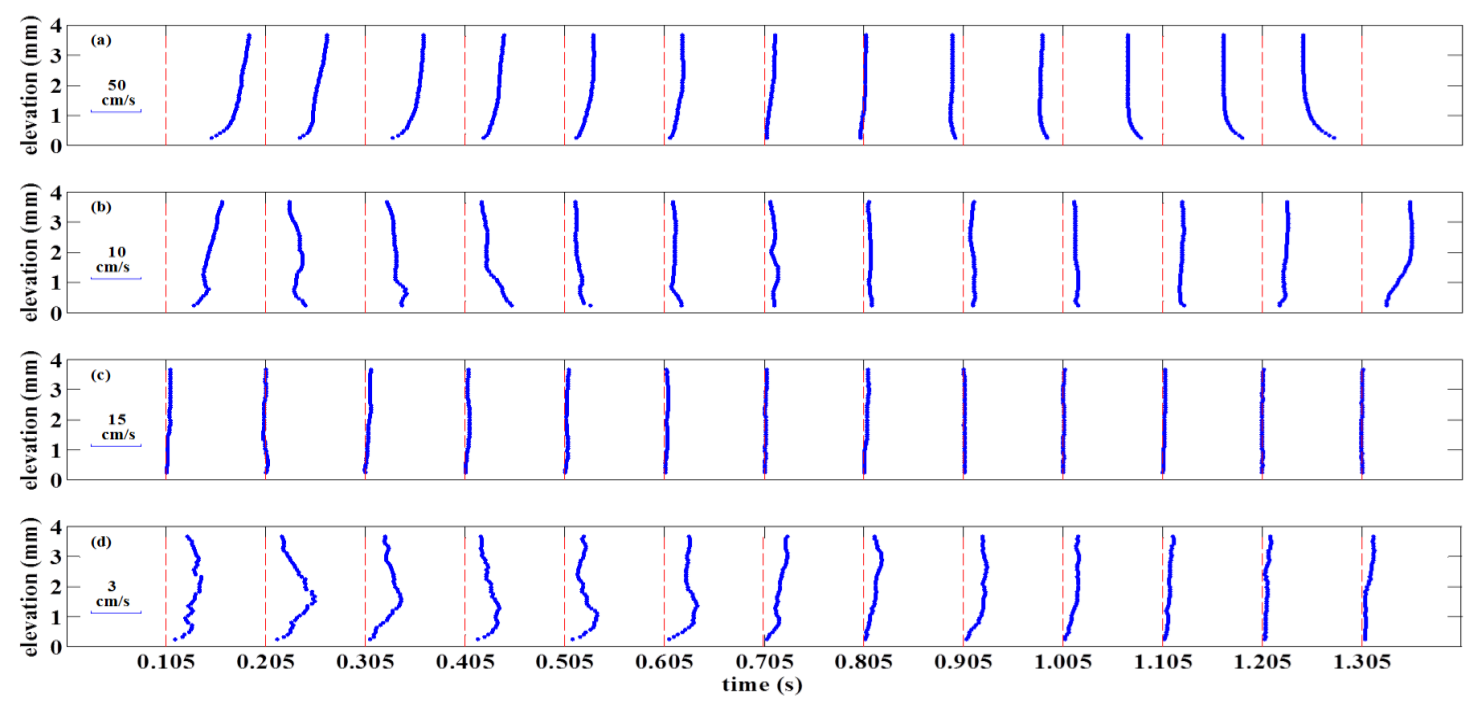

Fig.4 Vertical profiles of: (a) time-averaged $u$, (b) rms of $u$, (c) time-averaged $w$, (b) rms of $w$.

turbulence in the inertial subrange. Finally the measured velocities were band-pass filtered with a 5 $\mathrm{Hz}$ low limit cut-off frequency to remove the mean velocity and a $30 \mathrm{~Hz}$ high limit cut-off frequency to remove high frequency noise.

\section{RESULTS}

\section{(1) Velocity profiles}

Example time series of the velocity profiles averaged over $0.1 \mathrm{~s}$ in $x$ and $z$ directions are presented in Fig.4 (a) and Fig.4 (c), respectively. The time when the uprush water depth reaches $4 \mathrm{~mm}$ is defined as $t=0$. The last timing is when backwash water depth decreases to $4 \mathrm{~mm}$. The bed-parallel velocity profiles in the lower part are different from those presented in the previous studies (e.g. O'Donoghue ${ }^{2)}$ et al), whose lowest measurement point is higher above the bed. Their velocity profiles are generally closer to being depth-uniform. However, the velocity profiles of this study show the existence of logarithmic layer during the uprush and backwash stages as seen in Fig.4 (a). The bed-normal velocity is much smaller than the bed-parallel velocity as seen in Fig.4 (c), with a mean value close to zero.

\section{(2) Turbulence intensity}

Turbulence velocity is considered as a potential driver of sediment suspension in swash zone. The turbulence intensity was evaluated by taking the root mean square of the turbulent velocity over $0.1 \mathrm{~s}$ in this study. Time series of turbulence intensity profiles in $x$ and $z$ directions are presented in Fig.4 (b) and Fig.4 (d), respectively. It is demonstrated in Fig.4 that the turbulence intensity is in general proportional to the mean velocity. This tendency is very obvious in the initial stage of the uprush and the final stage of backwash. In the stage of flow reversal, the turbulence intensity is low due to the small magnitude of velocity and its gradient. The turbulence generated by the bore collapse is transported onto the beach and meanwhile the turbulence intensity generated by the high velocity gradient near the bed is transported upwards. The two sources of the turbulence intensity forms the peak close to the bed in the early uprush stage. However, the turbulence intensity in the upper layer is larger than that in the lower layer in the final stage of backwash. This looks strange and needs further discussion. One likely reason is that the high velocity and velocity gradient produce the higher turbulence intensity in the upper layer. As reported by O'Donoghue ${ }^{2}$ et al., although there is no bore influence during the late backwash, the flow processes in this stage is still not understood very well due to the high velocity and gradients. The turbulence intensity in Fig.4 (b) is relatively negligible compared to that in Fig.4 (d) (different scales were used in the two figures). The bed-normal turbulence intensity shows a similar distribution to the bed-parallel turbulence intensity.

\section{(3) Bed shear stress}

Reliable estimation of bed shear stress is of vital importance to understand the sediment transport process in swash zone. Although several methods have been attempted in many studies (e.g. O'Donoghue ${ }^{2)}$ et al., Shimozono ${ }^{7}$ et al., Yuan ${ }^{8}$ et al.), there is no generally accepted method of bed shear stress estimation in the swash zone because of the complicated flow conditions. Yuan ${ }^{8)}$ et al. concluded that the log-law method is considered to yield 


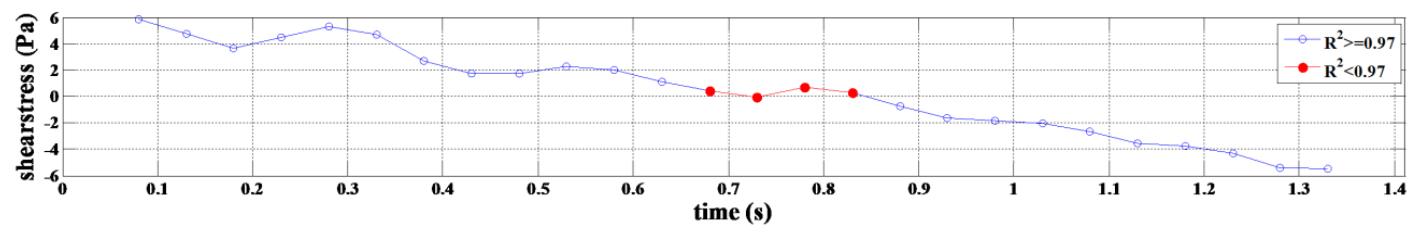

Fig.5 Estimated bed shear stress using log-law method.

relatively reasonable bed shear stress estimate; the Reynolds stress method underestimates the magnitude due to the missed small eddies (higher frequency turbulence). It also has a problem of uncertainty on determination of separation frequency to obtain the $u^{\prime}$ and $w^{\prime}$ as described in section 2.3, which are the key factors when applying the Reynolds stress method. Moreover, the log-law method is extensively used, making it possible to check and compare the results with previous results. Therefore, the log-law method was chosen in this study. The upper limit of the logarithmic velocity layer was identified in such a way that the best log fit among measurement points below was achieved.

Fig. 5 shows the time series of the bed shear stress calculated using the velocities averaged over $0.05 \mathrm{~s}$. The fit was accepted on the condition that three or more measurement points were used and the square of the correlation coefficient between measured data and fit exceeded 0.97. The bed shear stress shows a similar trend to previous studies: it is highest when bore arrives and decreases as the flow decelerates and increases again as the flow accelerate during the backwash; in the stage around flow reversal, the log-law fails since the flow here is laminar.

\section{CONCLUSIONS}

The velocity distribution within the bottom boundary layer in the bore-driven swash zone on impermeable steep slope was measured using a high-resolution PIV system. No previous study under the similar experiment conditions with such high resolution was found. The following are the main conclusions from this study.

i) The velocity profiles are similar to the previous studies. As expected, the high velocity with great gradients in the initial stage of uprush and the final stage of backwash are measured.

ii) The high turbulence intensity in the initial stage of uprush is supposed to be the sum of bore-generated turbulence associated with bore collapse and the bed-generated turbulence due to the high velocity gradients. The high turbulence intensity in the backwash is supposed to be the bed-generated turbulence. The source of the high turbulence intensity in the final stage of backwash is still unclear but it is supposed to be induced by the very high velocity gradients since the water depth is very thin.

iii) Estimates of bed shear stress based on log-law method reveal that the maximum uprush bed shear stress is larger than the maximum backwash bed shear stress.

The consistency of the velocity profiles between the current study and the previous studies demonstrates the reliability of the high-resolution PIV system. However, the conclusions of the current study are based on the detailed measurements within the boundary layer with high resolution and therefore the reliability should be greater than previous studies, which is a major highlight of this study. The repeatability of the experimental facility will be improved in the future study. Then by employing the advanced PIV system and ensemble average method, better insights into to swash zone boundary layer characteristics are expected.

\section{REFERENCES}

1) Cowen, E.A., Sou, I.M., Liu, P.L.F. and Raubenheimer, B.: Particle Image Velocimetry Measurements Within a Laboratory-Generated Swash Zone, J. En. Mech., Vol.129, pp.1119-1129, 2006.

2) O'Donoghue, T., D. Pokrajac and L. J. Hondebrink.: Laboratory and numerical study of dambreak-generated swash on impermeable slopes, Coastal Engineering, Vol. 57, pp. 513-530, 2010.

3) Shavit, U., R.J. Lowe and J.V. Steinbuck.: Intensity capping: a simple method to improve cross-correlation PIV results, Exp Fluids, Vol. 42, pp. 225-240, 2006.

4) Westerweel J, Dabiri D, and Gharib M.: The Effect of a discrete window offset on the accuracy of cross-correlation analysis of digital PIV recordings, Exp Fluids, Vol. 23, pp. 20-28, 1977.

5) Liu, Philip L-F., Yong Sung Park, and Edwin A. Cowen.: Boundary layer flow and bed shear stress under a solitary wave, J. Fluid Mech., Vol. 574, pp. 449-463, 2007.

6) Westerweel, J., Scarano, F.: Universal outlier detection for PIV data, Exp Fluids, Vol. 39, pp. 1096-1100, 2005.

7) Shimozono Takenori, Akio Okayasu, and Teppei Mishima.: On the bottom shear stress during long wave runup and backwash, Coastal Engineering Proceedings, 1(32): currents. 47, 2011.

8) Yuan J, Madsen O S.: Experimental study of turbulent oscillatory boundary layers in an oscillating water tunnel, Coastal Engineering, Vol. 89, pp. 63-84, 2014. 